\title{
New Quasi-Exactly Solvable Hamiltonians in Two Dimensions
}

Artemio González-López ${ }^{\S}$

Departamento de Física Teórica II

Universidad Complutense

28040 Madrid, Spain

\author{
Niky Kamran 9 \\ Department of Mathematics \\ McGill University \\ Montréal, Québec, Canada H3A 2K6
}

Peter J. Olver ${ }^{\dagger \ddagger}$

Department of Mathematics

University of Maryland

College Park, MD, U.S.A. 20742

\begin{abstract}
The purpose of this communication is to report on some recent progress in the problem of constructing quasi-exactly solvable Hamiltonians on two-dimensional Riemannian manifolds, [5].
\end{abstract}

The spectral problems of non-relativistic quantum mechanics fall within two general categories. In the first one, we have the small number of so-called exactly solvable problems, that is Schrödinger operators whose entire spectrum can be determined by algebraic methods. The simplest example of such a problem is given by the harmonic oscillator. In the second category, we have the Schrödinger operators whose complete spectrum cannot be computed exactly, but only approximated numerically at the very best.

Over the past decade, there has been a fair amount of interest in trying to construct physically significant systems which may not be exactly solvable, but for which part of the spectrum can be computed exactly by algebraic methods. In the early 1980's, Alhassid, Gürsey, Iachello, Levine and collaborators, $[\mathbf{1}],[\mathbf{1 0}]$, introduced the concept of a "spectrum generating algebra" to construct models for complicated molecules whose point spectrum could be analyzed algebraically. Independently, Turbiner, Ushveridze, Shifman and their collaborators were led to define a class of spectral problems which they called "quasi-exactly solvable", [14], [16], [12], and which we now describe.

A Schrödinger operator (or Hamiltonian)

$$
H=-\frac{1}{2} \sum_{i=1}^{d}\left(\frac{\partial}{\partial x^{i}}\right)^{2}+V\left(x^{1}, \ldots, x^{d}\right)
$$

(or, more generally, an arbitrary second-order linear differential operator) in $d$-dimensional Euclidean space is said to be Lie-algebraic if it is expressible as a bilinear combination

$$
\sum_{a, b=1}^{r} C_{a b} J^{a} J^{b}+\sum_{a=1}^{r} C_{a} J^{a}+C_{0}
$$

$\S$ Supported in Part by DGICYT Grant PS 89-0011.

I Supported in Part by an NSERC Grant.

$\dagger$ Supported in Part by NSF Grant DMS 92-04192.

$\ddagger$ On leave from School of Mathematics, University of Minnesota, Minneapolis, Minnesota, U.S.A. 55455. 
with constant coefficients $C_{a b}, C_{a}, C_{0}$ of first-order differential operators

$$
J^{a}=\sum_{i=1}^{d} \xi^{a i}(x) \frac{\partial}{\partial x^{i}}+\eta^{a}(x), \quad 1 \leq a \leq r,
$$

spanning a finite-dimensional Lie algebra $\mathfrak{g}$. If, in addition, $\mathfrak{g}$ admits a finite-dimensional module $\mathcal{N}$ of smooth functions, then $H$ is said to be quasi-exactly solvable. Obviously, if $\mathcal{N}$ is $k$-dimensional then one can obtain $k$ eigenvalues (counting multiplicities) of the Schrödinger operator $H$ with their corresponding linearly independent eigenfunctions by computing the spectrum of the finite-dimensional linear operator obtained by restriction of $H$ to the $k$-dimensional vector space $\mathcal{N}$. Finally, if the eigenfunctions of $H$ obtainable by the procedure just outlined turn out to be square integrable (i.e. if $\mathcal{N} \subset L^{2}\left(\mathbb{R}^{n}\right)$ ), and thus represent true bound states of the system, we shall say that $H$ is normalizable. The Lie algebra $\mathfrak{g}$ is to be thought of as a "hidden" symmetry algebra for the quasi-exactly solvable problem, whose presence underlies the partial solvability of the spectral problem. Notice, however, that $\mathfrak{g}$ is not a symmetry of $H$ in the traditional sense, since $H$ is not required to commute with the generators $J^{a}, 1 \leq a \leq r$, of $\mathfrak{g}$.

The class of all quasi-exactly solvable second-order linear differential operators $T(x)$ in $\mathbb{R}^{d}$ is easily seen to be invariant under the mapping

$$
T(x) \mapsto \bar{T}(z)=e^{\sigma(x)} T(x) e^{-\sigma(x)}, \quad z=\phi(x),
$$

where $\phi$ is a diffeomorphism. The transformation (1) has the key property of preserving the spectral problem associated to the second-order differential operator $T(x)$, i.e. if $\psi(x)$ is an eigenfunction of $T(x)$ with eigenvalue $\lambda$ then the "rescaled" function $\bar{\psi}(z)=e^{\sigma(x)} \psi(x)$ is an eigenfunction of $\bar{T}(z)$ with the same eigenvalue. Thus (1) defines a natural equivalence relation amongst quasi-exactly solvable second-order differential operators. (However, that $\bar{\psi}(z)$ need not be square integrable; see [4] for a thorough discussion of this problem in the one-dimensional case.)

The general procedure to be followed in order to classify all quasi-exactly solvable Hamiltonians under the equivalence relation (1) - a refinement of Levine's [10] original problem of classifying Lie-algebraic Schrödinger operators, which, in view of the above remarks, seems more interesting for the applications - is quite clear in principle, although the difficulties involved in implementing it in practice are enormous. See [5], and references therein, for an account of the present status of this problem.

The solution of the equivalence problem for second-order differential operators is relatively straightforward, [9], [3], and it leads to an essential distinction between the onedimensional and the higher-dimensional cases. Indeed, in one dimension every second-order differential operator is locally equivalent under (1) to a Schrödinger operator up to a sign, whereas this is no longer true in more than one dimension. The conditions under which a second-order differential operator is equivalent to a Schrödinger operator are quite stringent, $c f$. [5], but they suggest a natural simplification which might still be of interest for the applications, in view of recently uncovered connections with quantum chaos, [2], conformal field theory, $[\mathbf{1 1}],[\mathbf{8}]$, and the theory of orthogonal polynomials, [15]. Namely, we can enlarge the focus of our study from Schrödinger operators in Euclidean space to Schrödinger operators

$$
H=-\frac{1}{2} \sum_{i, j=1}^{d} g^{i j}(x) \nabla_{i} \nabla_{j}+V(x) \equiv-\frac{1}{2} \triangle+V(x)
$$


defined on arbitrary smooth Riemannian manifolds. Here, the functions $g^{i j}(x), 1 \leq i, j \leq$ $d$, are the contravariants components of a Riemannian metric $g, \nabla$ is the covariant derivative operator associated to the metric, and $\triangle$ is its corresponding Laplace-Beltrami operator.

A complete classification and list of normal forms for one-dimensional quasi-exactly solvable spectral problems is available, $[\mathbf{1 4}],[\mathbf{1 6}]$. In contrast, only a few special examples of quasi-exactly solvable problems in two dimensions have appeared in the literature to date, $[\mathbf{1 3}]$, all of which are constructed using only the compact "hidden" symmetry algebras $\mathfrak{s u}(3), \mathfrak{s u}(2) \oplus \mathfrak{s u}(2)$ or $\mathfrak{s o}(3)$. However, we know that there is a wide array of additional equivalence classes of Lie algebras of differential operators in two variables (admitting a finite-dimensional module of smooth functions), [6], [7], each of which can be used to construct new examples of two-dimensional quasi-exactly solvable spectral problems. A wide variety of interesting examples are given in [5], where particular emphasis is put on using non-compact "hidden" symmetry algebras to obtain quasi-exactly solvable twodimensional Hamiltonians. We shall content ourselves here with presenting two examples of new quasi-exactly solvable Hamiltonians in two dimensions, referring again the interested reader to [5] for further examples and details.

Consider, in the first place, the Lie algebra $\mathfrak{g} \cong \mathfrak{s l}(2) \oplus \mathfrak{s l}(2)$ spanned by the first-order differential operators

$$
J^{1}=\partial_{x}, \quad J^{2}=\partial_{y}, \quad J^{3}=x \partial_{x}, \quad J^{4}=y \partial_{y}, \quad J^{5}=x^{2} \partial_{x}-n x, \quad J^{6}=y^{2} \partial_{y}-m y,
$$

where the parameters $n$ and $m$ are restricted to taking positive integer values. The subspace $\mathcal{N}$ of the polynomials of degree no higher than $n$ in $x$ and $m$ in $y$ is then a $\mathfrak{g}$-module. One can then show that the Hamiltonian (2) with Riemannian metric and potential given by

$$
\begin{aligned}
g^{11}= & \left(1+x^{2}\right)\left(2+x^{2}\right), \quad g^{12}=\left(1+x^{2}\right)\left(1+y^{2}\right), \quad g^{22}=\left(1+y^{2}\right)\left(2+y^{2}\right) \\
8 V= & -y^{2}-\frac{(1+2 n)(3+2 n)}{1+x^{2}}-\frac{(1+2 m)(3+2 m)}{1+y^{2}} \\
& -\frac{17+12 y^{2}-y^{4}+2 x y\left(6+5 y^{2}\right)}{3+x^{2}+y^{2}}+\frac{5(3+2 x y)\left(1+y^{2}\right)\left(2+y^{2}\right)}{\left(3+x^{2}+y^{2}\right)^{2}}
\end{aligned}
$$

is normalizable and quasi-exactly solvable with respect to $\mathfrak{g}$.

Finally, let $\mathfrak{g}$ be the Lie algebra spanned by the first-order differential operators

$$
J^{1}=\partial_{x}, \quad J^{2}=\partial_{y}, \quad J^{3}=x \partial_{x}, \quad J^{4}=x \partial_{y}, \quad J^{5}=y \partial_{y}, \quad J^{6}=x^{2} \partial_{x}+r x y \partial_{y}-n x,
$$

and

$$
J^{6+i}=x^{i+1} \partial_{y}, \quad 1 \leq i \leq r-1,
$$

if $r>1, n$ being a positive integer. This non-compact Lie algebra, which is isomorphic to the semidirect product of $\mathfrak{g l}(2, \mathbb{R})$ with a $(r+1)$-dimensional abelian ideal, admits the finitedimensional module $\mathcal{N}$ spanned by the monomials $x^{i} y^{j}$ with $i+r j \leq n$. The Hamiltonian (2) with

$$
\begin{aligned}
g^{11} & =A x^{2}+B, \quad g^{12}=(1+m) A x y, \quad g^{22}=\nu\left(A x^{2}+B\right)^{m}+(1+m)^{2} A y^{2} \\
V & =-\frac{1}{2} \frac{A B \nu(1+m)^{2} \lambda(1+\lambda)\left(A x^{2}+B\right)^{m}}{\nu\left(A x^{2}+B\right)^{1+m}+A B(1+m)^{2} y^{2}}, \quad A, B, \nu>0, \mathbb{N} \ni m \leq r \neq 2(m+1),
\end{aligned}
$$


is shown in [5] to be normalizable and quasi-exactly solvable with respect to $\mathfrak{g}$, provided that the parameter $\lambda$ is large enough. Let us remark that the metric $g$ in this case has constant negative Gaussian curvature $\kappa=-A$. Furthermore, since the potential $V$ does not depend on the cohomology parameter $n$, the above Hamiltonian is exactly solvable in the sense of [14]. Notice, lastly, that the potential is also independent of $r$. We have thus constructed an exactly solvable Hamiltonian associated to an infinite number of inequivalent Lie algebras of arbitrarily large dimension $r+5$.

\section{References}

[1] Alhassid, Y., Gürsey, F., and Iachello, F., Group theory approach to scattering. II. The euclidean connection, Ann. Phys. 167 (1986), 181-200.

[2] Balazs, N.L., and Voros, A., Chaos on the pseudosphere, Physics Reports 143 (1986), 109-240.

[3] Cotton, É, Sur les invariants différentiels de quelques équations linéaires aux dérivées partielles du second ordre, Ann. École Norm. 17 (1900), 211-244.

[4] González-López, A., Kamran, N., and Olver, P.J., Normalizability of one-dimensional quasi-exactly solvable Schrödinger operators, Comm. Math. Phys., to appear.

[5] González-López, A., Kamran, N., and Olver, P.J., New quasi-exactly solvable Hamiltonians in two dimensions, preprint, Univ. of Minnesota, 1992.

[6] González-López, A., Kamran, N., and Olver, P.J., Lie algebras of differential operators in two complex variables, American J. Math., to appear.

[7] González-López, A., Kamran, N., and Olver, P.J., Quasi-exactly solvable Lie algebras of first order differential operators in two complex variables, J. Phys. A 24 (1991), 3995-4008.

[8] Gorsky, A., Relationship between exactly solvable and quasi-exactly solvable versions of quantum mechanics with conformal block equations in 2D theories, JETP Lett. 54 (1991), 289-292.

[9] Kamran, N., and Olver, P.J., Equivalence of differential operators, SIAM J. Math. Anal. 20 (1989), 1172-1185.

[10] Levine, R.D., Lie algebraic approach to molecular structure and dynamics, in: Mathematical Frontiers in Computational Chemical Physics, D.G. Truhlar, ed., IMA Volumes in Mathematics and its Applications, Vol. 15, Springer-Verlag, New York, 1988, pp. 245-261.

[11] Morozov, A.Y., Perelomov, A.M., Rosly, A.A., Shifman, M.A. and Turbiner, A.V., Quasi-exactly solvable quantal problems: one-dimensional analogue of rational conformal field theories, Int. J. Mod. Phys. 5 (1990), 803-832.

[12] Shifman, M.A., New findings in quantum mechanics (partial algebraization of the spectral problem), Int. J. Mod. Phys. A 126 (1989), 2897-2952.

[13] Shifman, M.A. and Turbiner, A.V., Quantal problems with partial algebraization of the spectrum, Commun. Math. Phys. 126 (1989), 347-365.

[14] Turbiner, A.V., Quasi-exactly solvable problems and $\mathfrak{s l}(2)$ algebra, Commun. Math. Phys. 118 (1988), 467-474.

[15] Turbiner, A.V., Lie algebraic approach to the theory of polynomial solutions, preprint, CERN, CPT-92/P.2679, 1992.

[16] Ushveridze, A.G., Quasi-exactly solvable models in quantum mechanics, Sov. J. Part. Nucl. 20 (1989), 504-528. 